\title{
EFECTO DE LA APLICACIÓN DE CITOQUININAS SOBRE LA REGENERACIÓN DE BROTES in vitro DE Rhodophiala rhodolirion*
}

\section{EFFECT OF CYTOKININS ON THE in vitro BUD REGENERATION OF Rhodophiala rhodolirion.}

\author{
Seemann, P.', Jara, G.', Muñoz, M.', Riegel, R.', Schiappacasse, F. ${ }^{2}$, Peñailillo, P. ${ }^{2}$ y Vico, V. ${ }^{2}$ \\ 'Instituto de Producción y Sanidad Vegetal, Universidad Austral de Chile, Valdivia, Chile. \\ E-mail: pseemann@uach.cl \\ ${ }^{2}$ Departamento de Horticultura, Universidad de Talca, Talca, Chile.
}

\section{INTRODUCCIÓN}

Rhodophiala rhodolirion es una planta ornamental geófita nativa, con bulbo tunicado, de $1-4,1 \mathrm{~cm}$ de diámetro. Habita en las laderas secas y asoleadas de la Cordillera de los Andes y de la Costa entre la V-VII Región. Presenta una inflorescencia con una sola flor solitaria grande, rosado-carmín, que la hace muy atractiva ornamentalmente. Debido a que su propagación vegetativa en forma natural no ha sido eficiente se han debido estudiar otras alternativas de propagación, como la micropropagación, la cual ha sido aplicada exitosamente en una gran cantidad de geófitas ornamentales. Debido a esto la presente investigación plantea promover la formación in vitro de brotes de Rhodophiala rhodolirion bajo medios inductivos con diferentes concentraciones y tipos de citoquininas.

\section{MATERIAL Y MÉTODOS}

El ensayo se estableció con dos bulbos de Rhodophiala rhodolirion colectados en la localidad de Pichuante, VII región. Las escamas fueron sembradas en medio MS, con macrosales reducidas al $75 \%$, adicionado con agar $(0,8 \%)$, carbón activado $(0,5 \%)$, sacarosa $(3 \%)$ y ácido naftalen acético, ANA $\left(0,1 \mathrm{mg} \mathrm{L}^{-1}\right)$. El medio fue suplementado con 6-Benzilaminopurina (BAP), 2-isopentiladenina (2iP), Tidiazurón
(TDZ) y kinetina (KIN) en concentraciones de 0,5 y $1,0 \mathrm{mg} \mathrm{L}^{-1}$, además de los testigos (T1 y T2) sin reguladores de crecimiento $(0 / 0)$ o solo con ácido naftalen acético $(0,1 / 0)$

Se aislaron secciones de escamas dobles de cada uno de los bulbos las que fueron desinfectadas con etanol $70 \%$ e hipoclorito de sodio $1 \%$. Se obtuvieron 131 secciones del bulbo 1 y 69 del bulbo $2(\mathrm{n}=200)$. Cada sección o "explante" se sembró en frascos individuales con $10 \mathrm{~mL}$ de medio de cultivo, incubadas durante 120 días en cámara con fotoperíodo de 16 horas luz, con un flujo de fotones fotosinteticamente activos de $50 \mu \mathrm{mo} \mathrm{m}^{-2} \mathrm{~s}^{-1}$ y $23^{\circ} \mathrm{C}$, para finalmente realizar las siguientes evaluaciones: Número y longitud de brotes con microbulbillos y porcentaje de brotación

\section{RESULTADOS}

Los resultados indican que la sobrevivencia y brotación alcanzan un valor promedio del 26 y $21 \%$ respectivamente. La brotación fue más adecuada en el medio con $0,1 \mathrm{mg} \mathrm{L}^{-1}$ de ANA y $0,5 \mathrm{mg} \mathrm{L} \mathrm{L}^{-1}$ de BAP, sin diferencias estadísticamente significativas con las citoquininas restantes. Por otra parte se presentó un rendimiento promedio de 28 microbulbillos por bulbo, lo cual refleja la baja capacidad de regeneración que presenta Rhodophiala rhodolirion a partir de material vegetativo (Cuadro 1). 
Cuadro 1. Parámetros de sobrevivencia y brotación en Rhodophiala rhodolirion.

Table 1. Survival parameters and budding in Rhodophalia rhodolirion.

\begin{tabular}{llcc}
\hline Trat. & \multicolumn{1}{c}{$\begin{array}{c}\text { ANA/Citoquinina } \\
\text { (mg/L) }\end{array}$} & $\begin{array}{c}\% \\
\text { Sobrevivencia }\end{array}$ & $\begin{array}{c}\% \\
\text { Brotación }\end{array}$ \\
\hline $\mathbf{1}$ & $\mathbf{0 / 0}$ (T1) & 15 & 5 \\
$\mathbf{2}$ & $\mathbf{0 , 1 / 0 , 5}$ BAP & 30 & 55 \\
$\mathbf{3}$ & $\mathbf{0 , 1 / 1 , 0 ~ B A P ~}$ & 30 & 20 \\
$\mathbf{4}$ & $\mathbf{0 , 1 / 0 , 5 ~ 2 i P}$ & 25 & 15 \\
$\mathbf{5}$ & $\mathbf{0 , 1 / 1 , 0 ~ 2 i P}$ & 35 & 30 \\
$\mathbf{6}$ & $\mathbf{0 , 1 / 0 , 5} \mathbf{~ K I N ~}$ & 26 & 16 \\
$\mathbf{7}$ & $\mathbf{0 , 1 / 1 , 0 ~ K I N ~}$ & 38 & 9 \\
$\mathbf{8}$ & $\mathbf{0 , 1 / 0 , 5}$ TDZ & 25 & 5 \\
$\mathbf{9}$ & $\mathbf{0 , 1 / 1 , 0 ~ T D Z}$ & 20 & 20 \\
$\mathbf{1 0}$ & $\mathbf{0 , 1 / 0}($ T2) & 15 & 35 \\
& Promedio & $\mathbf{2 6}$ & $\mathbf{2 1}$ \\
\hline
\end{tabular}

Cuadro 2. Número y longitud de brotes en Rhodophiala rhodolirion.

Table 2. Number and length of Rhodophalia rhodolirion buds.

\begin{tabular}{|c|c|c|}
\hline $\begin{array}{c}\text { Conc. } \\
\text { ANA/Citoquinina } \\
(\mathrm{mg} / \mathrm{L})\end{array}$ & $\begin{array}{l}\text { No de } \\
\text { brotes }\end{array}$ & $\begin{array}{c}\text { Long. de } \\
\text { brotes }(\mathrm{cm})\end{array}$ \\
\hline $0 / 0(\mathrm{~T} 1)$ & $0,05 \mathrm{bc}$ & $0,05 \mathrm{~b}$ \\
\hline $0,1 / 0,5$ BAP & $0,55 a$ & $0,3 a$ \\
\hline $0,1 / 1,0$ BAP & $0,2 a b c$ & $0,05 \mathrm{~b}$ \\
\hline $0,1 / 0,52 \mathrm{iP}$ & $0,15 a b c$ & $0,12 a b$ \\
\hline $0,1 / 1,02 \mathrm{iP}$ & $0,3 a b c$ & $0,15 a b$ \\
\hline $0,1 / 0,5 \mathrm{KIN}$ & $0,16 a b$ & $0,13 \mathrm{ab}$ \\
\hline $0,1 / 1,0 \mathrm{KIN}$ & 0,09 abc & $0,07 a b$ \\
\hline $0,1 / 0,5 \mathrm{TDZ}$ & $0,05 \mathrm{c}$ & $0,15 \mathrm{ab}$ \\
\hline $0,1 / 1,0$ TDZ & $0,2 a b c$ & $0,015 a b$ \\
\hline $0,1 / 0(\mathrm{~T} 2)$ & 0,35 abc & $0,08 \mathrm{ab}$ \\
\hline
\end{tabular}

\section{CONCLUSIONES}

- La sobrevivencia y brotación presentó valores promedio de un 26 y $21 \%$ respectivamente.

- La mejor inducción de brotes se logró en el medio de cultivo adicionado con $0,1 \mathrm{mg} \mathrm{L}^{-1}$ de
ANA y $0,5 \mathrm{mg} \mathrm{L}^{-1}$ de BAP, sin diferencias estadísticamente significativas con las otras citoquininas.

-Se obtuvo un rendimiento promedio de $28 \mathrm{mi}$ crobulbillos por bulbo. 